EPJ Web of Conferences 87,02015 (2015)

DOI: $10.1051 /$ epjconf/ 20158702015

(C) Owned by the authors, published by EDP Sciences, 2015

\title{
Study of Synergetic Effect of X2 and X3 EC Wave in KSTAR
}

\author{
Y.S. Bae ${ }^{1, a}$, J. Decker ${ }^{2}$, J.H. Jeong ${ }^{1}$, K.D. Lee ${ }^{1}$ \\ ${ }^{1}$ National Fusion Research Institute, Daejeon 305-333, Korea \\ ${ }^{2}$ Association Euratom-CEA, DSM/IRFM, CEA Cadarache, 13108 St Paul lez Durance, France
}

\begin{abstract}
An experimental study of the X-mode absorption at the second and the third harmonic frequencies has been performed in KSTAR tokamak. The X2 EC frequency is $110 \mathrm{GHz}$ and the X3 EC frequency is 170 $\mathrm{GHz}$ at the nominal KSTAR operating toroidal magnetic field. From the 1-D model of the synergetic effect, the $\mathrm{X} 3$ cold resonance should lie at low field side with the X2 cold resonance at the high field side to meet the condition which both $\mathrm{X} 2$ and $\mathrm{X} 3 \mathrm{EC}$ waves interact with the same resonant electron at the same radial position. However, $170 \mathrm{GHz}$ X3 cold resonance lies at the high field side with distance of $54 \mathrm{~mm}$ from the X2 cold resonance position in KSTAR. This paper presents the study of the synergetic effect of X3 absorption by the $\mathrm{X} 2 \mathrm{ECCD}$ with a scheme of two beam target positions at the same flux surface by scanning the poloidal and toroidal beam injection angles to enhance X3 absorption even in the low temperature plasma in KSTAR. For this study, the3D relativistic ray/tracing and Fokker-Planck codes C3PO/LUKE is used for the quantitative prediction of its synergetic effect. The $\mathrm{C} 3 \mathrm{PO} / \mathrm{LUKE}$ codes are appropriate for $\mathrm{X} 2$ and $\mathrm{X} 3$ synergy calculations as the distribution function can be calculated with either one or both waves affecting the absorption rate of each other. This paper also introduces the future ECH system upgrade plan.
\end{abstract}

\section{Introduction}

The KSTAR ECRH systems have two different frequencies, $110 \mathrm{GHz}$ and $170 \mathrm{GHz}$ which are second and third harmonic frequency resonant to the operation toroidal magnetic field of $2 \mathrm{~T}$ which is selected for the high beta operation in KSTAR second phase. Figure 1 shows the X-mode cut-off and the ray tracing from the low-field side launch of each EC wave for $110 \mathrm{GHz}$ and $170 \mathrm{GHz}$ assuming the parabolic density profile with central density of $5 \times 10^{19} \mathrm{~m}^{-3}$.

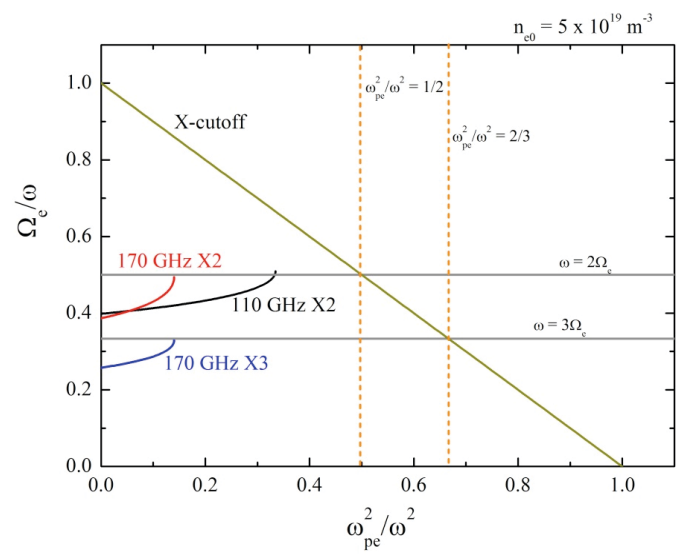

Figure 1. The X-mode cut-off and low-field side launch of 110 $\mathrm{GHz}$ and $170 \mathrm{GHz}$ EC wave in KSTAR for the central density, $\mathrm{n}_{\mathrm{e} 0}=5 \times 10^{19} \mathrm{~m}^{-3}$.

\footnotetext{
${ }^{\mathrm{a}}$ Corresponding author: ysbae@nfri.re.kr
}

The $110 \mathrm{GHz}$ and $170 \mathrm{GHz}$ X-mode cut-off density at the second harmonic resonance position is $7.47 \times 10^{19} \mathrm{~m}^{-3}$ and $1.78 \times 10^{20} \mathrm{~m}^{-3}$, respectively.

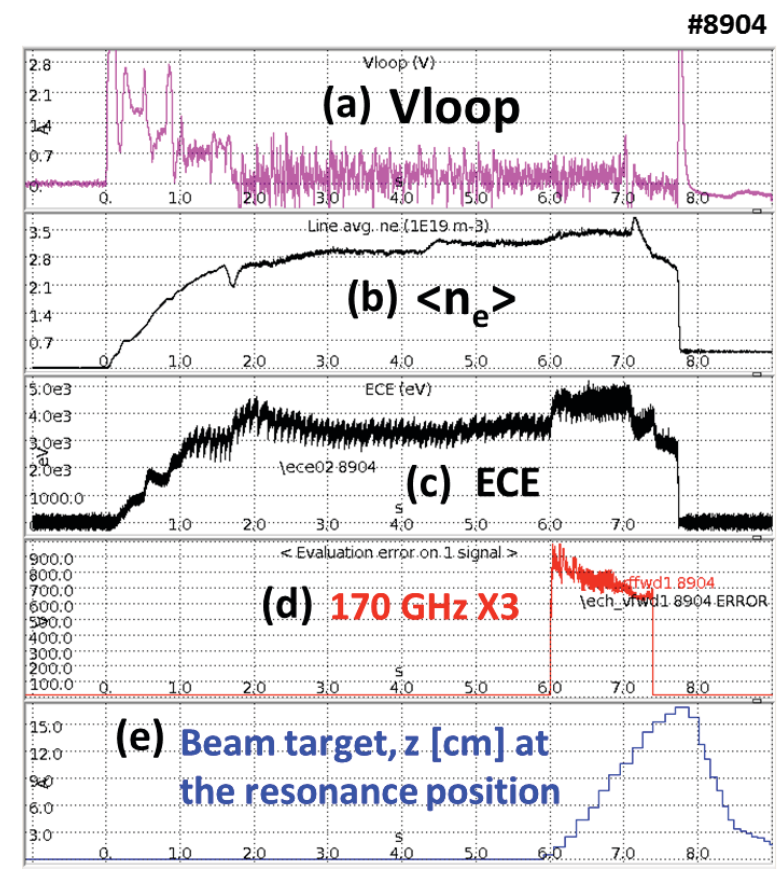

Figure 2. The time traces of shot no. 8904 for third harmonic EC heating experiment. 
The electron cyclotron (EC) wave at the third harmonic extraordinary (X3) mode is optically thin with the lower The electron cyclotron (EC) wave at the third harmonic extraordinary (X3) mode is optically thin with the lower absorption rate than the $\mathrm{EC}$ wave at the second harmonic extraordinary (X2) mode by a factor of $k_{\mathrm{b}} T_{\mathrm{e}} / \mathrm{mc}^{2}$ hence the damage of wall by non-absorbed EC power through the resonance layer is critical issue in KSTAR operation. Therefore, the high electron temperature plasma is favorable to have better X3 EC wave absorption. Figures 2 shows the efficient $170 \mathrm{GHz}$ X3 EC heating observed in NBI-heated high temperature plasma. The line average density and temperature at the center is $3.5 \times 10^{19} / \mathrm{m}^{3}$ and $3.5 \mathrm{keV}$, respectively, before $170 \mathrm{GHz} \mathrm{EC}$ wave injection. The line average density begins to increase before the EC power injection, the loop voltage is not changed owing to the EC heating effect. The temperature increased in ECE channel looking the plasma center by about $30 \%$ during EC power on. In this particular shot, the launcher steering mirror is also moved from the on-axis to off-axis as seen in fig. 2 (d). Figure 3 shows the temperature profile measurement by ECE radiometer channels corresponding to the experimental data in fig. 2. For the temperature profile measurement at given time, each ECE channel data is averaged every 1-ms interval time. The temperature profile changes to the centrally peaked profile by central heating in the middle of EC power pulse. At $\mathrm{t}=7.2 \mathrm{~s}$ when EC beam vertical target position at the resonance location is moved from the core to the edge, $\mathrm{z} \sim 12 \mathrm{~cm}$ during the EC power pulse, the core temperature drops back to the initial temperature before the EC power pulse. But, the temperature profile is broadened with increased temperature at off-axis.

But $\mathrm{TcV}$ experiment $[1,2]$ showed the enhanced $\mathrm{X} 3$ absorption through the synergetic effect of the X3 EC wave absorption when X2 electron cyclotron current drive (ECCD) is applied together. According to TcV experiments, there is evidence that some of the injected $\mathrm{X} 3 \mathrm{EC}$ power is absorbed by supra-thermal electrons in an energetic tail created by the X2 ECCD preheating.

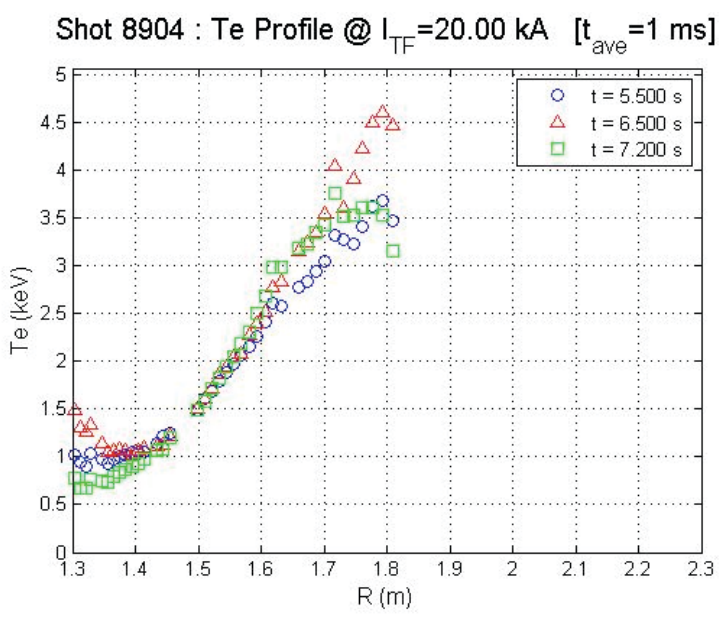

Figure 3. The electron temperature profile measured by ECE radiometer channels corresponding to the data in fig. 2 .
The simplified 1-D model of the synergetic effect between X2 and X3 is just relativistic Doppler-shifted resonance relation:

$$
\begin{gathered}
\omega-k_{\|} v_{\|}=n \Omega(R) / \gamma \\
\gamma=\left[1+p^{2} /\left(m^{2} c^{2}\right)\right]^{1 / 2}
\end{gathered}
$$

Since the finite absorption occurs for $p_{\perp}>0$

$$
n \Omega(R) / \omega=\left(1-N_{\|}{ }^{2}\right)^{1 / 2}
$$

Letting $N_{\|}=\sin \phi$

Corresponding single particle resonant kinetic energy

$$
\begin{aligned}
& \mathrm{E}_{\mathrm{X} 2 \mathrm{ECCD}}=(\gamma-1) m_{\mathrm{e}} c^{2} \approx 1 / 2 \sin ^{2} \phi\left(m_{\mathrm{e}} c^{2}\right) \\
& \mathrm{E}_{\mathrm{X} 3 \mathrm{ECH}}\left(N_{\|}=0\right)=\left(R_{\mathrm{X} 3} / R-1\right) m_{\mathrm{e}} c^{2}
\end{aligned}
$$

The single particle resonant kinetic energy and toroidal injection angle as a function of major radius are plotted in fig. 4 for $110 \mathrm{GHz}$ and $170 \mathrm{GHz}$. The intersection between the single particle resonant energies for the $\mathrm{X} 2$ and $\mathrm{X} 3$ waves defines the same radial position where both waves can interact with electrons of the same kinetic energy, but there is no intersection for $110 \mathrm{GHz}$ and $170 \mathrm{GHz}$ in KSTAR like TcV case [1]. However, there is overlap resonance zone in 2-D as seen in raytracing and quasi-linear Fokker-Planck calculation in fig. 3 in next section.

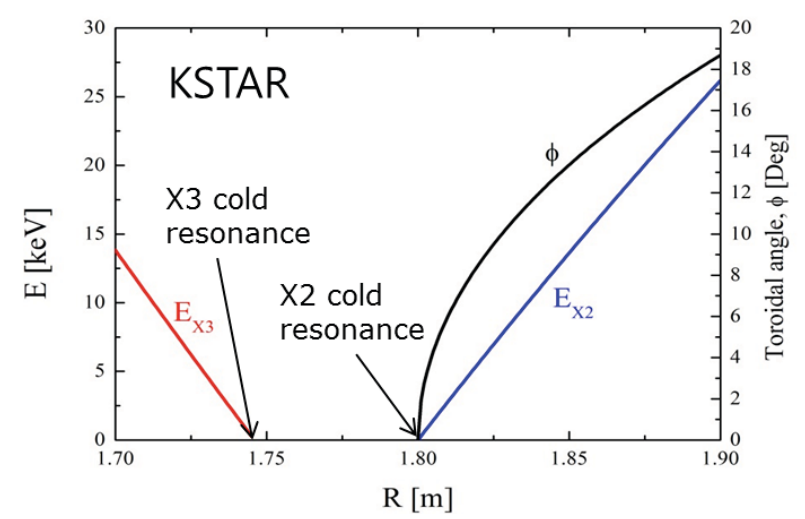

Figure 4. The 1-D model for single particle resonant kinetic energies $\mathrm{E}$ of two $110 \mathrm{GHz}$ X2 and $170 \mathrm{GHz}$ X3 EC wave as a function of radial distance of major axis, R. The toroidal incidence angle $\phi$ is also plotted.

For the $140 \mathrm{GHz}$ and $170 \mathrm{GHz} \mathrm{EC}$ waves, there is an intersection point in 1-D model as seen fig. 5. However, it requires high resonant kinetic energy and toroidal injection angle. The $140 \mathrm{GHz}$ is other frequency for the new ECH system using 105/140 GHz two frequency gyrotron.

\section{Simulation results}

\subsection{Fokker-Planck solver LUKE}

\section{Synergy effect}


Resonant interaction between electrons and RF waves can lead to a significant distortion of the e- $\mathrm{f}(\mathrm{t})$ function, which in turns affects the damping rate and CD efficiency of the RF waves. This effect cannot be described by linear modelling of EC heating and $\mathrm{CD}$. Instead, it requires solving the 3D Fokker-Planck (FP) equation with a quasilinear (QL) treatment of thee wave-particle interaction as provided by 3D FP solver. Here we used LUKE [3] for FP solver and C3PO [4] for ray-tracing.

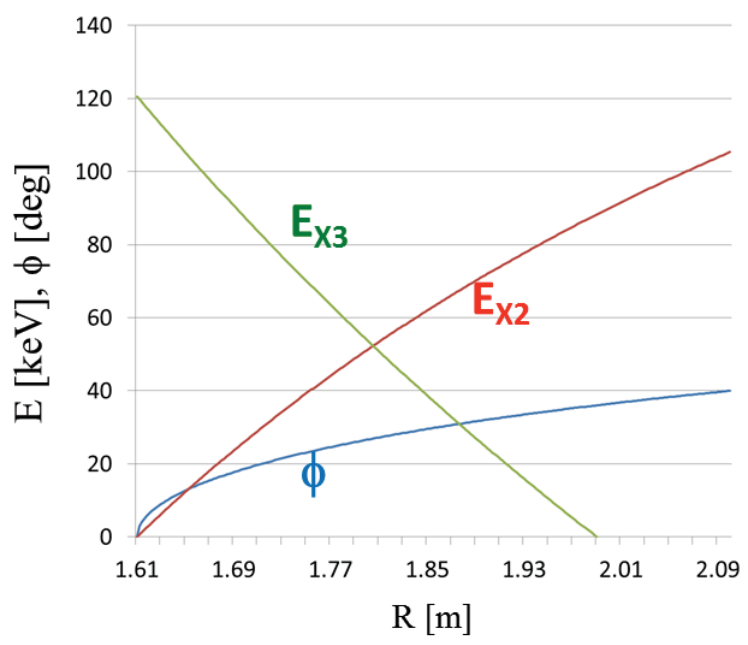

Figure 5. The 1-D model for single particle resonant kinetic energies $\mathrm{E}$ of two $140 \mathrm{GHz} \mathrm{X} 2$ and $170 \mathrm{GHz} \mathrm{X} 3 \mathrm{EC}$ wave as a function of radial distance of major axis, R. The toroidal incidence angle $\phi$ is also plotted.

\subsection{Simulation results}

Figure 6 shows the ray tracing and power deposition profile of $110 \mathrm{GHz}$ X2 and $170 \mathrm{GHz}$ X3 EC waves for parabolic temperature and density profiles with $\mathrm{Te}(0)=$ $3.2 \mathrm{keV}$, and ne $(0)=3.5 \times 10^{19} / \mathrm{m}^{3}$ for typical KSTAR Lmode discharge. The synergy effect is expected by the resonance overlap zone through the same flux surface between two EC waves as seen fig. 6 .
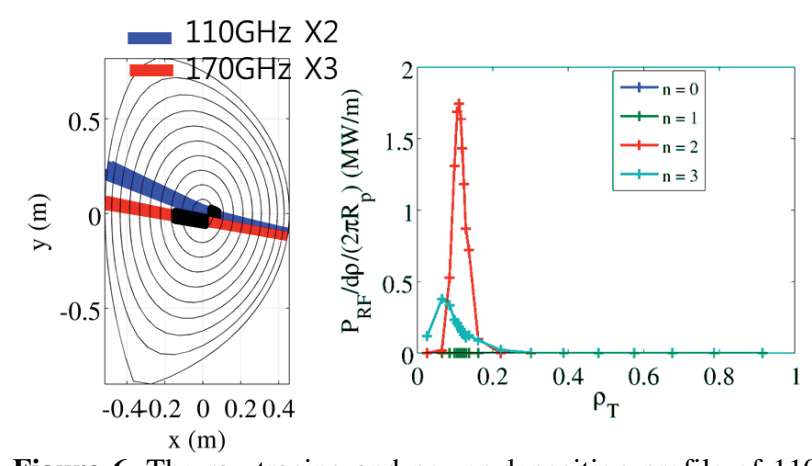

Figure 6. The ray tracing and power deposition profile of 110 $\mathrm{GHz}$ X2 and $170 \mathrm{GHz}$ X3 EC wave at toroidal magnetic field of $2 \mathrm{~T}$.

To investigate the synergy effect on single-pass absorption of X3 wave, the X2 EC wave injection angles are varied. Figure 7 shows the single-pass absorption ratio of $\mathrm{X} 3 \mathrm{EC}$ wave for the $\mathrm{X} 2 \mathrm{EC}$ wave injection angles.
The maximum absorption ratio is obtained in certain range of poloidal and toroidal injection angles. Without $\mathrm{X} 2$ EC wave injection, the absorption ratio of $\mathrm{X} 3 \mathrm{EC}$ wave is about $45.8 \%$. When X2 EC wave is injected, the absorption ratio of $\mathrm{X} 3 \mathrm{EC}$ wave increased a little. The maximum increase is about $2 \%$ for certain $\mathrm{X} 2 \mathrm{EC}$ wave injection angle. The synergetic effect is maximized for the poloidal injection angle $\theta>10 \mathrm{deg}$ at the toroidal injection angle $6 \mathrm{deg}<\phi<9 \mathrm{deg}$. The maximum absorption ratio of X3 EC wave has nearly linear dependence on $\mathrm{X} 2 \mathrm{EC}$ power as seen fig. 8 .
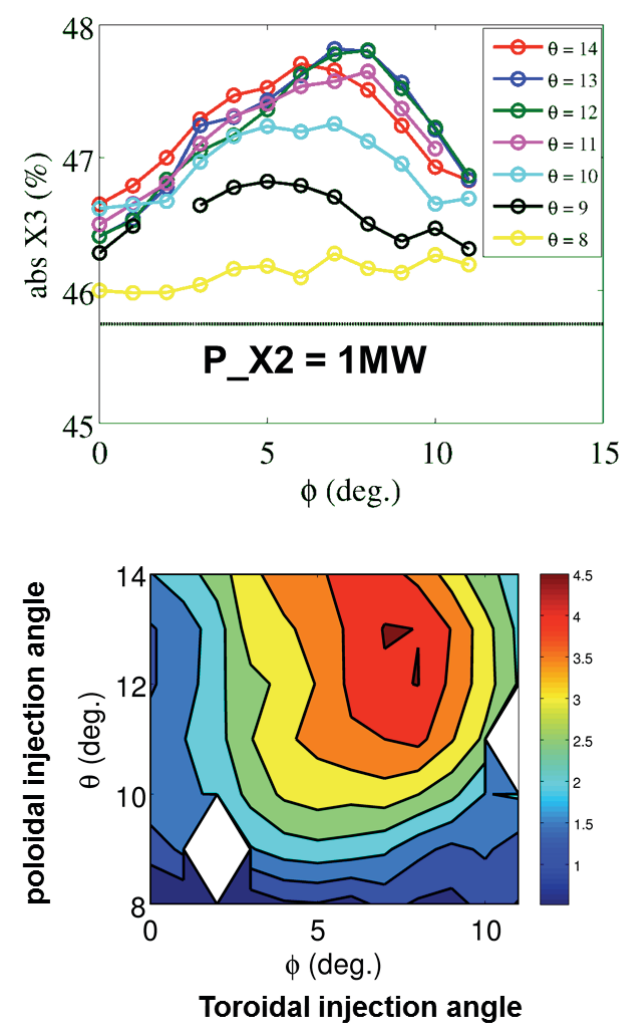

Figure 7. The absorption ratio of $\mathrm{X} 3 \mathrm{EC}$ wave versus $\mathrm{X} 2 \mathrm{EC}$ wave injection angles with fixed X2 EC power of $1 \mathrm{MW}$. The solid horizontal line in top figure depicts the absorption ratio of $\mathrm{X} 3 \mathrm{EC}$ wave without X2 EC wave injection.

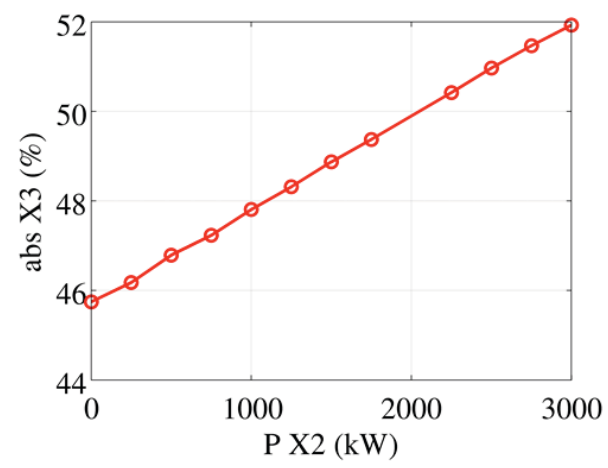

Figure 8. The absorption ratio of $\mathrm{X} 3 \mathrm{EC}$ wave versus the $\mathrm{X} 2$ EC power.

\section{$4 \mathrm{ECH}$ upgrade plan}

ECH/ECCD system should have a capability of long pulse operation to achieve the high performance, steady 
state tokamak operation. While the EC current drive is predicted to be a capable of producing elevated $q_{\text {min }}$ plasmas with reactor relevant condition, high $T_{\mathrm{e}} / T_{\mathrm{i}}$. Due to the limited total heating power, these advanced operation modes are predicted to be accessed at the reduced toroidal magnetic field, $B_{\mathrm{t}} \sim 2 \mathrm{~T}$. Therefore, KSTAR has a plan to add the new $3 \mathrm{MW}$ EC systems with total three $1 \mathrm{MW}, 300 \mathrm{~s}, 105 / 140 \mathrm{GHz}$ twofrequency gyrotrons during KSTAR 2nd phase. Figure 9 shows the layout design view of new three gyrotrons with an existing $170 \mathrm{GHz}, 1 \mathrm{MW}$ ECH gyrotron. The twofrequency gyrotron will be a GYCOM gyrotron with specifications of maximum duration of $300 \mathrm{~s}$ at the RF output power at gyrotron window; $950 \mathrm{~kW}$ at $140 \mathrm{GHz}$ (main frequency) and $800 \mathrm{~kW}$ at $105 \mathrm{GHz}$, and the oscillation efficiency $>45 \%$ with depressed collector and diode gun.

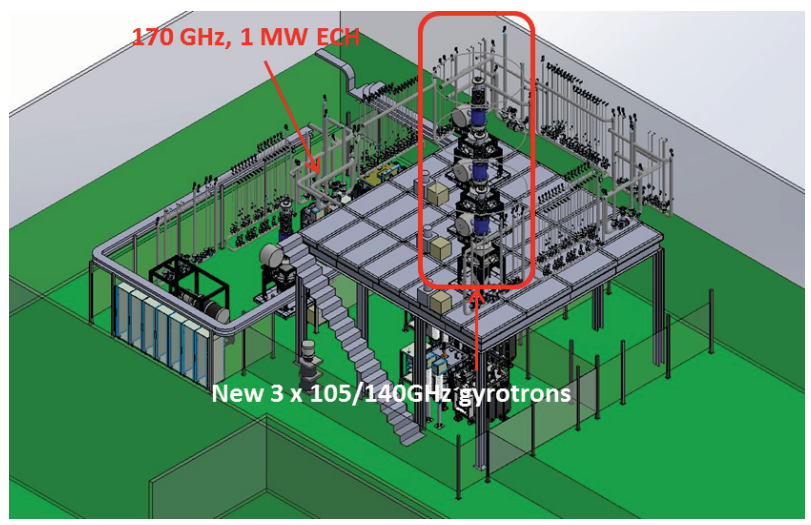

Figure 9. Layout design of new 105/140 GHz two-frequency ECH system.

\section{Summary}

$\mathrm{ECH}$ is known as very useful tool in fusion plasma physics research in tokamak and stellarator. Maximizing absorption of EC wave is important issue for the efficient electron heating and current drive at second and third harmonics. So, the use of synergetic effect between X2 and X3 EC waves is promising method. In KSTAR ECRH using two different frequencies of $110 \mathrm{GHz}$ and $170 \mathrm{GHz}$, preliminary LUKE calculation shows nearly linear dependence of $\mathrm{X} 3$ absorption ratio on $\mathrm{X} 2 \mathrm{EC}$ power and the optimum condition of $\mathrm{X} 3$ absorption ratio exists with certain range of X2 EC wave injection angles.

Future plan is LUKE simulation for synergetic effect between $140 \mathrm{GHz}$ X2 and $170 \mathrm{GHz}$ X3 wave. We have the experimental plan investigating $110 \mathrm{GHz}$ and 170 $\mathrm{GHz}$ synergetic effect through the same flux surface in high and low Te plasma conditions in 2014 KSTAR campaign. The X3 absorption ratio will be measured using X3 EC beam power modulation and diamagnetic loop signal with the relation of $\mathrm{P}_{\mathrm{abs}}=(\mathrm{dWp} / \mathrm{dt})_{\mathrm{ECH} \_\mathrm{ON}}-$ $(\mathrm{dWp} / \mathrm{dt})_{\mathrm{ECH} \_\mathrm{OFF}}$. Also, the evidence of synergetic effect will be investigated using newly installed HXR detector of fast electron bremsstrahlung emission and comparison between ECE and TS and soft x-ray.

\section{References}

1. S. Alberti, T.P. Goodman, M.A. Henderson, A Manini, J.-M. Moret, P. Gomez, P. Blanchard, S. Coda, O. Sauter, Y. Peysson, TCV Team, Nucl. Fusion 42 (2002) 42.

2. S. Gnesin, J. Decker, S. Coda, T.P. Goodman, Y. Peysson and D. Mazon, Plasma Phys. Control. Fusion 54 (2012) 035002.

3. J. Decker et al., Euratom-CEA report EUR-CEA-FC1736, 2004.

4. Y. Peysson, J. Decker, L. Morini and S. Coda, Plasma Phys. Control. Fusion 53124028 (2011). 